\title{
Perfil dos premiados em Olimpíadas de Informática e sua influência sobre a Educação em Computação
}

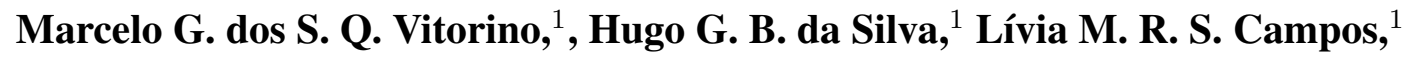 \\ Rohit Gheyi ${ }^{1}$ \\ ${ }^{1}$ Departamento de Sistemas e Computação \\ Universidade Federal de Campina Grande \\ Av. Aprígio Veloso, 882 - 58.109-970, Campina Grande, PB - Brasil \\ \{marcelo.vitorino, hugo.silva\}@ccc.ufcg.edu.br \\ \{livia,rohit\}@computacao.ufcg.edu.br
}

\begin{abstract}
Attending computer science competitions aims at developing skills that contribute to the students school life, improving their performance in other disciplines, and promoting interest in solving day-to-day problems with computer artifacts. However, little is known about the strategies used in the training of the laureate students. In this paper, we analyze data from 163 Informatics Olympics medalists from the Paraiba state in 2016 and 2017 using interviews and survey. The two studies aimed at identifying the main practices that contributed to the good performance of the medalists what could influence other students to improve their own performances in this kind of competition and, thus, expand their potentials in Informatics.
\end{abstract}

Resumo. A participação em competições de informática possibilita aos alunos o desenvolvimento de aptidões que os auxiliam na sua vida escolar, melhorando o seu desempenho em outras disciplinas, além de promover o interesse em resolver problemas do dia-a-dia com artifícios computacionais. Entretanto, pouco se sabe quais as estratégias utilizadas nos treinamentos dos alunos laureados. Neste trabalho foram analisados dados de 163 medalhistas de olimpíadas de informática realizadas na Paraíba (OPI) em 2016 e 2017 usando entrevistas e survey. Os dois estudos identificaram aspectos importantes das práticas utilizadas para o bom desempenho desses participantes na competição, podendo influenciar outros participantes de olimpíadas a melhorarem seus desempenhos e, assim, expandirem seus conhecimentos nesse contexto.

\section{Introdução}

As Olimpíadas Científicas aproximam os estudantes de determinadas áreas por meio de um sistema de competição saudável que, além de envolver o aprendizado técnico daquela área, pode desenvolver uma série de habilidades transversais tais como, trabalho em equipe, bons hábitos de estudo, conscientização ambiental, maior vínculo com a escola e professores, capacidade de resolver problemas, autoconfiança e autoestima [Rocha et al. 2016]. No Brasil, existem olimpíadas em diversas áreas de conhecimento (Matemática, Física, Ciências, História, Astronomia, Robótica, Informática, dentre outras) e para diferentes níveis educacionais. 
VII Congresso Brasileiro de Informática na Educação (CBIE 2018)

Anais do XXIX Simpósio Brasileiro de Informática na Educação (SBIE 2018)

A Olimpíada Paraibana de Informática (OPI) [OPI 2018] vem sendo realizada na Paraíba desde o ano de 2008 abrangendo estudantes de diversas cidades do estado. Tem como objetivo despertar nos estudantes o interesse em computação, imprescindível na formação básica escolar atualmente, além de permitir que os competidores possam conhecer de forma mais abrangente a carreira na área. Para os alunos do ensino superior, a OPI visa estimular os alunos a aprimorarem e aprofundarem o conhecimento em Programação e Algoritmos. Além disso, a olimpíada almeja uma melhor preparação e o despertar do interesse de alunos paraibanos para as competições nacionais e internacionais de programação, como a Olimpíada Brasileira de Informática [OBI 2018], e a ACM International Collegiate Programming Contest ACM/ICPC [ICPC 2018].

É possível encontrar na literatura relato de experiências sobre os benefícios trazidos pela presença de estudantes em olimpíadas de informática. A participação em competições de informática possibilita ao aluno o desenvolvimento de aptidões que os auxiliam na sua vida escolar, melhorando o seu desempenho em outras disciplinas, além de promover o interesse em resolver problemas do dia-a-dia com artifícios computacionais [de Barros et al. 2009].

Nascimento et al. (2007) declaram que a participação de alunos de um curso técnico no Pará ajudou a reduzir a descrença na auto-capacidade de realização, desenvolver a autonomia, além de melhorar a atenção e concentração em determinadas aulas. Santos et al. (2015) afirmam que a execução de um projeto de extensão sobre a Olimpíada de Informática em uma cidade da Paraíba, contribuiu para a inclusão social de muitos estudantes, incluindo setor público e privado, sendo algo inovador, gratuito e que auxiliou o ensino e didática dos professores em sala de aula. Segundo Duarte et al. (2010) a imersão dos alunos de um curso superior de computação na Paraíba em competições de informática tem provocado o aumento da quantidade de alunos que gostam de programar.

Ainda no âmbito da educação superior, o relato de alguns participantes da OPI reforça o impacto positivo que tal experiência trouxe para sua vida escolar, melhorando o desempenho nas disciplinas do curso e abrindo oportunidades para projetos extraclasses. Além disso, eles relataram que a participação em olimpíadas ajudou na preparação para entrevistas de emprego e estágio, e até levando-os a obterem êxito nas mesmas. Entretanto, pouco se sabe sobre as estratégias usadas pelos alunos premiados.

O presente artigo tem como objetivo analisar, usando entrevistas e surveys, o perfil de 163 participantes medalhistas de olimpíadas de informática realizadas na Paraíba (OPI) em 2016 e 2017, por categoria. Os dois estudos buscam identificar e caracterizar situações específicas, motivações e práticas utilizadas para o bom desempenho desses participantes na competição, podendo influenciar outros participantes de olimpíadas a melhorarem seus desempenhos e, assim, expandirem seus conhecimentos nesse contexto. É importante salientar que este trabalho visa além de alcançar os competidores das olimpíadas, estender conclusões que possam servir como análise para grupos acadêmicos motivados na preparação dos estudantes para as competições, a partir de experiências dos premiados.

Este artigo está estruturado da seguinte forma. Na Seção 2 tem-se a apresentação dos materiais e métodos usados nos dois estudos, enquanto na Seção 3 apresentam-se os principais resultados dos estudos. Por fim, na Seção 4 apresentam-se as principais conclusões obtidas. 
VII Congresso Brasileiro de Informática na Educação (CBIE 2018)

Anais do XXIX Simpósio Brasileiro de Informática na Educação (SBIE 2018)

\section{Materiais e Métodos}

Para o estudo realizado, combinamos métodos empíricos de pesquisa distintos e complementares, incluindo entrevistas semiestruturadas e surveys (formulários). Tais métodos nos permitiram conhecer o pensamento e comportamento dos medalhistas da OPI nas edições de 2016 e 2017, considerando os resultados pelas categorias definidas na competição, quais sejam: Iniciação I e II (para alunos do $1^{\circ}$ ao $8^{\circ}$ do ensino fundamental - explora questões de raciocínio lógico e analítico); Programação (para alunos $9^{\circ}$ ano do ensino fundamental e alunos do ensino médio - explora questões de programação envolvendo técnicas, estruturas de dados e algoritmos básico); Avançado Júnior e Sênior (para alunos de cursos superiores até o primeiro ano do Mestrado - explora questões de programação avançadas).

\subsection{Questões de Pesquisa}

A fim de alcançarmos os objetivos aqui propostos, elaboramos as seguintes questões de pesquisa:

- QP1: Quais as estratégias de preparação mais comuns entre os medalhistas?

- QP2: De onde vem o incentivo mais comum para os medalhistas?

- QP3: Existe um perfil que caracteriza os medalhistas antes de começarem a participar de olimpíadas de informática?

- QP4: Qual a relação dos medalhistas com a computação?

Apresentaremos os resultados observados para essas questões na Seção 3.

\subsection{Estratégias de Pesquisa}

A pesquisa foi realizada em duas etapas principais. Na primeira etapa (Estudo I) foram realizadas entrevistas semiestruturadas presenciais, com premiados da categoria Avançado (júnior e sênior) da OPI 2016, segundo o método Grounded Theory [Glaser and Strauss 1999]. Já a segunda etapa (Estudo II) foi composta por duas rodadas de entrevistas estruturadas, através de formulários, com os premiados de todas as categorias da OPI 2016 e 2017. Os principais materiais utilizados na pesquisa se encontram neste repositório de dados público. ${ }^{1}$

\subsection{Estudo I - Entrevistas Semiestruturadas}

Na primeira etapa do estudo, um conjunto de 7 medalhistas nas categorias Avançado Júnior e Avançado Sênior da OPI 2016 foi entrevistado. O critério usado para a ordem dos entrevistados foi agrupar de acordo com as categorias da competição e coletar uma amostra aleatória simples de cada uma destas categorias.

Para processamento e análise das entrevistas foi utilizado o método do Grounded Theory, que consiste em obter um conjunto de hipóteses conceituais integradas geradas sistematicamente para produzir uma teoria sobre uma área de interesse. De forma geral, pode ser entendido como um método para gerar uma teoria a partir dos dados analisados.

Foram elaboradas algumas perguntas iniciais para os entrevistados, com base nas questões de pesquisa definidas, mas, não se limitando as mesmas. As entrevistas foram realizadas individualmente e sempre com o mesmo entrevistador; todas foram gravadas e

\footnotetext{
${ }^{1}$ https://github.com/marcelovitorino/PerfilDosPremiados
} 
VII Congresso Brasileiro de Informática na Educação (CBIE 2018)

Anais do XXIX Simpósio Brasileiro de Informática na Educação (SBIE 2018)

posteriormente transcritas. As transcrições foram analisadas seguindo o método escolhido e utilizando os seguintes passos: codificação das respostas, análise de palavras-chave, organização destas em conceitos e categorias e a escrita de memos. Posteriormente foi gerado um documento final de coding.

O principal objetivo das entrevistas semiestruturadas foi fundamentar e melhorar a segunda fase do estudo, como descrito a seguir.

\subsection{Estudo II - Formulários}

Para segunda etapa do estudo foram realizadas entrevistas estruturadas através de formulários (surveys) que foram respondidos por 163 medalhistas da OPI entre 2016 e 2017.

As perguntas contidas nos formulários aplicados em 2016 e 2017, disponibilizados em versão impressa e online, levaram em consideração o resultado das entrevistas semiestruturadas, buscando explorar: dados gerais dos alunos, participação em Olimpíadas, interesse em computação/programação e planos futuros. Visando abranger uma quantidade maior de premiados, as coletas de dados, além de terem sido realizadas via e-mail, também foram aplicadas durante as cerimônias de premiação da OPI que ocorreram em 2016 e 2017.

Após a coleta de dados, utilizou-se o Python e R como linguagens para processamento dos dados e criação de visualizações. Os dados coletados foram analisados seguindo o processo Knowledge Discovery in Databases (KDD) [Fayyad et al. 1996], por meio das seguintes atividades:

- Seleção dos dados, advindos dos formulários, relevantes para a análise;

- Pré-processamento e limpeza dos dados, removendo valores nulos, por exemplo;

- Transformação dos dados, onde foi possível estruturá-los aplicando a formatação necessária para seu processamento e geração de visualizações;

- Interpretação/avaliação dos dados por meio de análise descritiva a partir das questões de pesquisa definidas (Seção 3).

A análise considerou os dados agrupados pelas três categorias da OPI, referenciadas neste estudo pelos nomes Iniciação, Intermediário e Avançado. A distribuição dos dados coletados por perfil está descrita na Tabela 1. As amostras coletadas para cada perfil não possuem o mesmo tamanho, logo, algum perfil pode estar mais bem representado do que outro. Além disso, a população da pesquisa inclui premiações de menções honrosas.

Tabela 1. Dados Coletados nos anos de 2016 e 2017.

\begin{tabular}{|r|r|r|r|}
\hline Perfil dos Premiados & Amostra & População & Proporção da Amostra \\
\hline Iniciação & 65 & 215 & $30,23 \%$ \\
\hline Intermediário & 28 & 63 & $44,44 \%$ \\
\hline Avançado & 70 & 147 & $47,61 \%$ \\
\hline Total & 163 & 425 & $38,35 \%$ \\
\hline
\end{tabular}

\section{Resultados e Discussão}

A partir da análise dos dados coletados em 2016, observou-se uma maior concordância de características entre premiados das diferentes categorias da OPI, sugerindo assim a 
VII Congresso Brasileiro de Informática na Educação (CBIE 2018)

Anais do XXIX Simpósio Brasileiro de Informática na Educação (SBIE 2018)

existência dos três perfis de premiados. O mesmo ocorreu para os dados coletados em 2017, permitindo relacionar os dados dos dois anos de estudo.

Os resultados serão apresentados em função das questões de pesquisa definidas na Seção 2.1, que ajudam a identificar relações nas estratégias de preparação dos premiados e estabelecer práticas determinantes para o bom desempenho nas competições, utilizando pontos pré-definidos.

\section{QP1: Quais as estratégias de preparação mais comuns entre os medalhistas?}

Havia uma questão nos formulários dos dois anos sobre as formas de preparação com as seguintes opções: "treinamento na escola/universidade", "estudei com amigos e/ou colegas", "estudei sozinho" e "não me preparei". Sendo que, em 2016 a questão foi de múltipla resposta (com associação com outra opção), e em 2017 a questão foi de única resposta (sem associação com outra opção).

Nos dois anos, considerando todos os perfis, a opção "não me preparei" não teve associação com outras opções. Além disso, para o Perfil Iniciação em 2016, mesmo com o questionário concitando a múltipla escolha, a maioria das respostas também não obtiveram associações. Tais resultados permitiram observar alguns padrões durante os dois anos, a saber: 1) "não me preparei" representou 17,79 \% da população total de dados coletados durante a pesquisa; 2) "estudei sozinho" e "treinamento na escola/universidade" foram as opções mais frequentes para os iniciantes.

Para estudar a relação dos perfis Intermediário e Avançado nos dois anos, considerou-se, primeiramente, os dados de 2016, que dão margem a associação entre as opções. Para tal ano foi feita uma análise utilizando grafos de co-ocorrências. Posteriormente, esses grafos foram interpretados em comparativo com os dados de 2017, que não possuíam associação. Nesse caso, percebeu-se que a principal relação de associação destacada em 2016 ("treinamento na escola/universidade" + "estudo sozinho") para os dois perfis, são as opções mais significativas também durante o ano de 2017, como exibido na Figura 1.

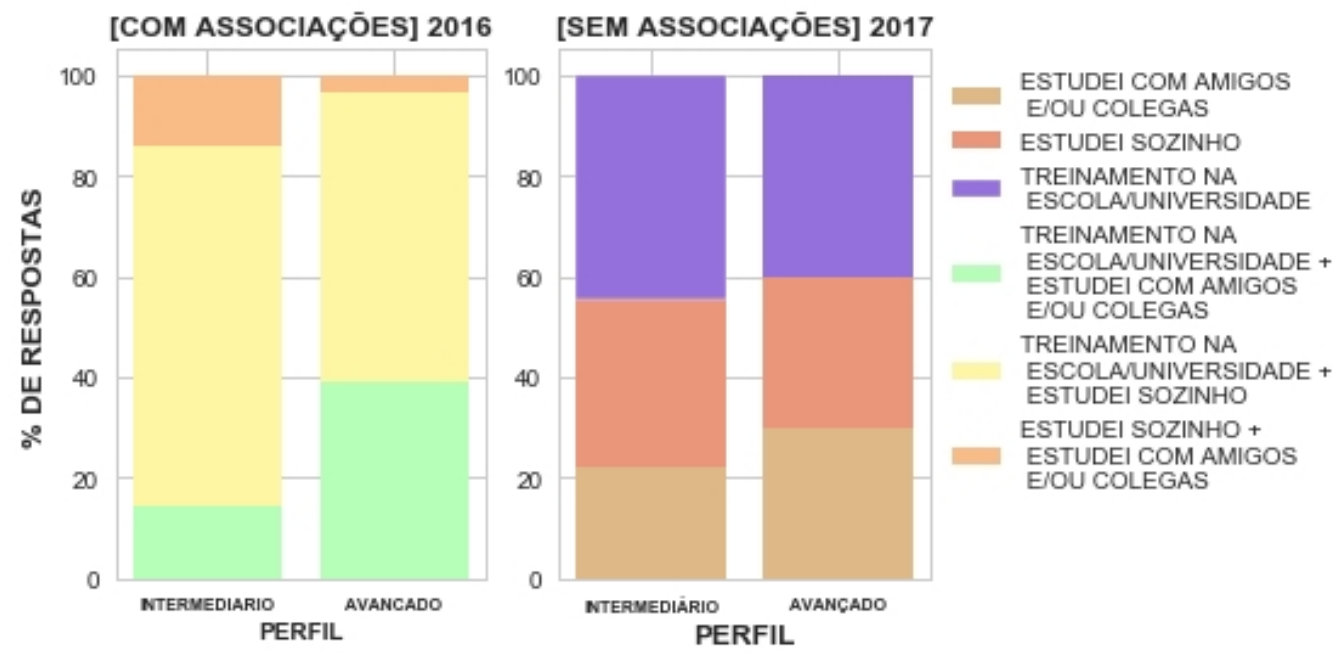

Figura 1. Formas de preparação

Em relação ao material utilizado, nos formulários dos dois anos questionou-se 
VII Congresso Brasileiro de Informática na Educação (CBIE 2018)

Anais do XXIX Simpósio Brasileiro de Informática na Educação (SBIE 2018)

sobre materiais didáticos e plataformas de treinamento mais comuns. Porém, em 2017 a questão era aberta e única, já em 2016, haviam questões separadas e ambas de múltipla escolha. Mesmo assim, observou-se que havia associações entre as opções. Além disso, para tratamento da questão única em 2017, foi feita uma extração em cada valor derivando valores materiais e plataformas como abordado em 2016. Os resultados são ilustrados na Figura 2.

Note que, para o Perfil Iniciação, as opções "material da internet", "provas de outras edições da olimpíada", e a plataforma URI Online Judge [Judge 2018] estão bem relacionadas. Já no Perfil Intermediário, a plataformas URI Online Judge é a que possui mais associações, e em particular, a associação entre URI Online Judge, Codeforces e material da internet é bem forte. O uso das plataformas foi ainda mais citado no Perfil Avançado onde se pode observar uma forte relação entre URI Online Judge, Codeforces e Spoj.
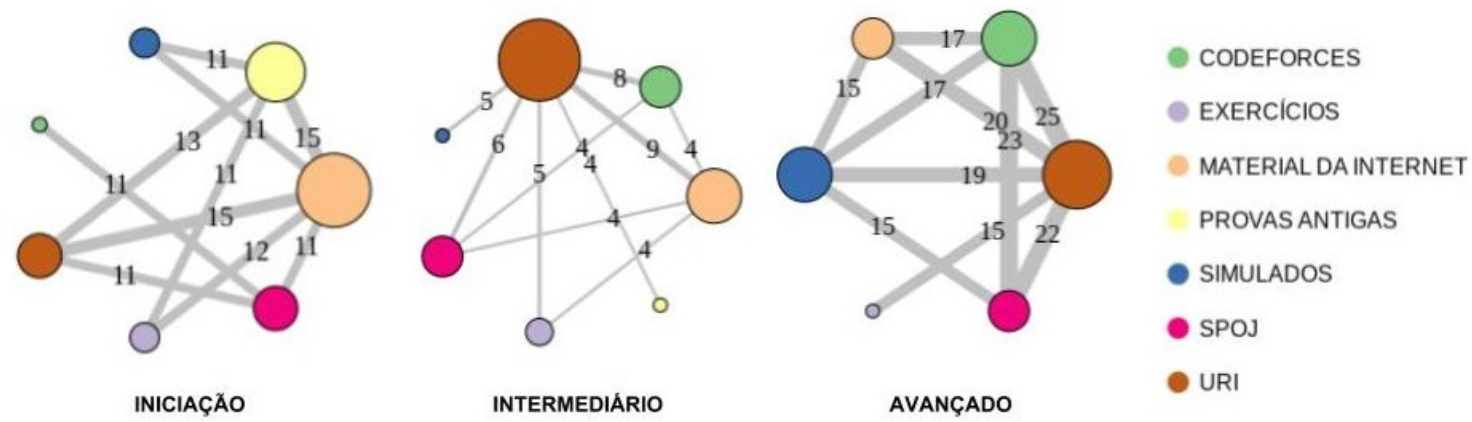

Figura 2. Plataformas e materiais utilizados.

Em relação ao tempo de estudo por semana dos premiados, utilizando a mediana como métrica, observou-se que o tempo mediano de estudo do Perfil Iniciação é de 2 horas, o do Perfil Intermediário de 3 horas, e o Perfil Avançado de 4,5 horas.

\section{QP2: De onde vem o incentivo mais comum para os medalhistas?}

O estudo buscou identificar de onde advém o incentivo mais comum para os medalhistas, no qual foi verificado que premiados do Perfil Avançado possuem como principais incentivadores os amigos e colegas de forma constante durante os anos da pesquisa. É importante salientar que tais premiados competem em equipe. Em contrapartida, esse é um aspecto que não é utilizado por premiados do Perfil Iniciação, para os mesmos incentivadores. Já para o Perfil Intermediário, sobretudo em 2017, a iniciativa própria é determinante para tais competidores (ver Figura 3).

\section{QP3: Existe um perfil que caracteriza os medalhistas antes de começarem a partici- par de olimpíadas de informática?}

Ao analisar essa característica, verificou-se que os premiados de todas as categorias costumam participar de olimpíadas científicas de outras áreas e que, de forma predominante, a participação nas olímpiadas de matemática se destaca para todos os perfis de medalhistas. Além disso, a atração pela resolução de problemas que envolvem raciocínio lógico e a intenção de melhorar as notas escolares são as justificativas dos premiados para suas participações nas olimpíadas de informática. 
VII Congresso Brasileiro de Informática na Educação (CBIE 2018)

Anais do XXIX Simpósio Brasileiro de Informática na Educação (SBIE 2018)
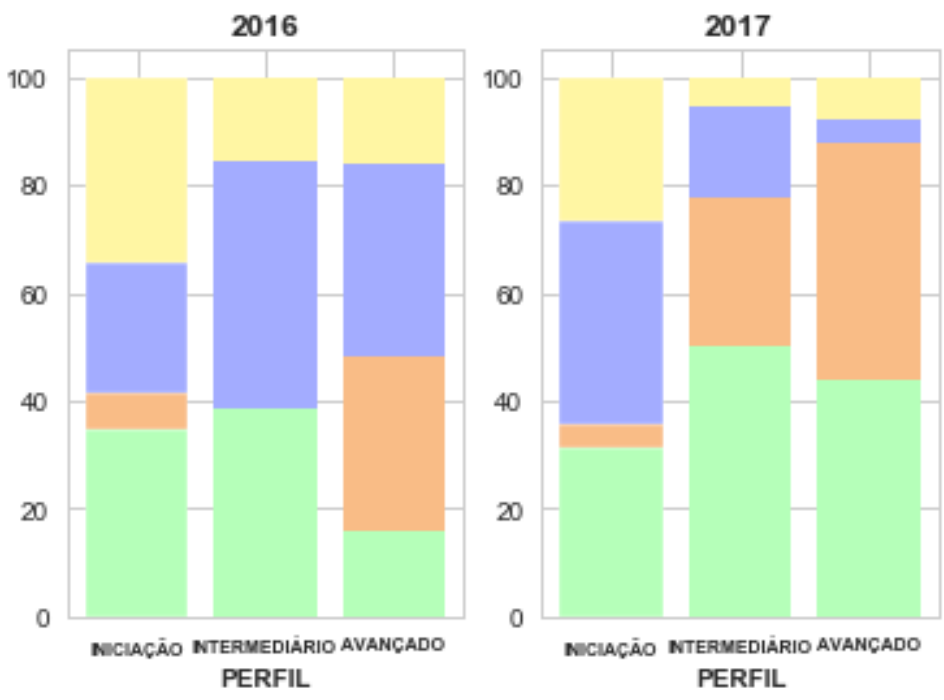

INICIATIVA PROPRIA

(EU MESMO!)

MEUS AMIGOS

E COLEGAS

MINHA ESCOLA

MINHA FAMILIA

Figura 3. Advento do incentivo para competir.

No ano de 2017, por exemplo, foi verificada que a disciplina de Matemática é a mais consensual, em relação ao melhor desempenho, entre os medalhistas dos perfis Iniciação e Intermediário (80,4\%), e que os premiados do Perfil Avançado possuem desempenho de destaque em disciplinas que envolvem Programação (76\%).

\section{QP4: Qual a relação dos medalhistas com a computação?}

Ao estudar a relação dos medalhistas com a computação, foi possível observar que a maioria dos premiados do Perfil Iniciação não sabem programar (76,9\%). Mas que $73,3 \%$ dos premiados que não sabem programar demonstram interesse em aprender programação.

Analisando o nível de conhecimento em programação dos premiados foi possível observar alguns padrões nos dois anos da pesquisa, como ilustrados na Figura 4. De forma geral, o nível cresce linearmente com o perfil no qual ele se enquadra.

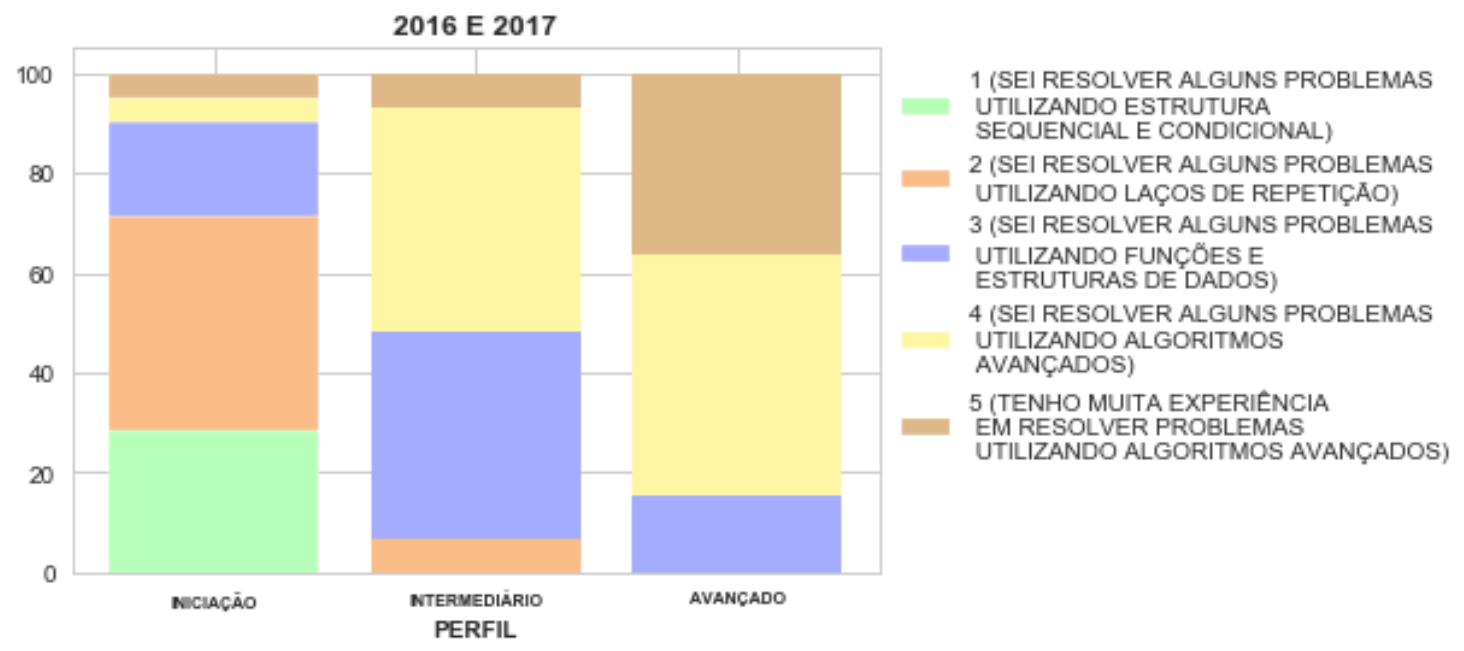

Figura 4. Nível de conhecimento em programação. 
VII Congresso Brasileiro de Informática na Educação (CBIE 2018)

Anais do XXIX Simpósio Brasileiro de Informática na Educação (SBIE 2018)

Ainda sob essa vertente, foi observado que as linguagens Python, Java e C/C++ são as mais comumentes utilizadas entre os medalhistas. Foi feito também um estudo com premiados do Perfil Intermediário, no ensino médio, e verificou-se que nos dois anos Computação é o curso de maior pretensão para prestar vestibular dentre os premiados analisados. Além disso, todos os entrevistados demonstram interesse em continuar competindo.

\subsection{Lições Aprendidas}

A forma de preparação é um fator essencial para o bom desempenho nas olimpíadas de informática, e está relacionada a aspectos individuais dos competidores que refletem no tipo de preparação escolhido.

A partir dos resultados alcançados com as respostas aos formulários com os medalhistas da OPI 2016 e 2017, foi possível estruturar aspectos essenciais para cada perfil de medalhista, como ilustrado na Tabela 2.

Tabela 2. Características determinantes dos premiados em cada categoria.

\begin{tabular}{|l|l|}
\hline $\begin{array}{l}\text { Perfil } \\
\text { de premiados }\end{array}$ & Aspectos de destaque \\
\hline Iniciação & $\begin{array}{l}\text { Utilização de provas antigas, da plataforma URI Online Judge } \\
\text { (para os que programam) e de materiais na internet; Participação } \\
\text { em treinamentos promovidos pela escola; Participação em outras } \\
\text { olimpíadas; Conhecimento em programação no nível de } \\
\text { Resolução de problemas utilizando laços de repetição (para os } \\
\text { que programam); Tempo mínimo de estudo por semana esperado } \\
\text { de 2h. }\end{array}$ \\
\hline Intermediário & $\begin{array}{l}\text { Utilização de plataformas de preparação como URI Online Judge } \\
\text { e Codeforces e materiais na internet; Participação em } \\
\text { treinamentos promovidos pela escola; Bom desempenho em } \\
\text { Matemática; Iniciativa própria para participar da OPI; } \\
\text { Conhecimento em programação no nível de Resolução de } \\
\text { problemas utilizando funços, estruturas de dados e alguns } \\
\text { algoritmos avançados; Tempo mínimo de estudo por semana } \\
\text { esperado de 3h. }\end{array}$ \\
\hline Avançado & $\begin{array}{l}\text { Utilização de simultâneas formas de preparação (treinamentos na } \\
\text { universidade, estudo sozinho, com amigos,etc) e de simultâneas } \\
\text { plataformas (URI Online Judge, SPOJ, Codeforces, dentre } \\
\text { outras); Incentivo de amigo para participar da OPI; Bom } \\
\text { desempenho em disciplinas de Programação; Certa experiência } \\
\text { em resolução de problemas utilizando algoritmos avançados; } \\
\text { Tempo mínimo de estudo por semana esperado de 4,5 horas. }\end{array}$ \\
\hline
\end{tabular}

Vale ressaltar que, treinamentos promovidos pela escola para seus estudantes são também citados em outros trabalhos envolvendo experiências exitosas com olimpíadas 
VII Congresso Brasileiro de Informática na Educação (CBIE 2018)

Anais do XXIX Simpósio Brasileiro de Informática na Educação (SBIE 2018)

de informática na educação básica [Kiryukhin 2010], [Burton 2008]. Além disso, é inegável que a participação em olimpíadas de informática contribui para a formação em computação e desenvolve um conjunto de habilidades nos participantes que delineiam um bom profissional, competências estas que ajudarão no desenvolvimento e sucesso do participante em qualquer ambiente de trabalho que escolha. [Kiryukhin 2010], [do Nascimento et al. 2007], [Duarte et al. 2010], [Dagiené 2010].

\subsection{Ameaças à Validade}

O Estudo I realizou entrevistas com um número pequeno de participantes. Mesmo sendo um número baixo, foi possível identificar uma tendência nas respostas, e ajudou na estruturação do Estudo II. O conjunto de lições aprendidas no Estudo II pode não ser um completo. Entretanto, um número maior de pessoas (163) foram consideradas nesse estudo para minimizar essa ameaça à validade do estudo.

\section{Conclusão}

Com a análise dos dados coletados, foi possível identificar, a partir do mapeamento com as questões de pesquisa, que o treinamento com a escola/universidade foi a forma de preparação mais utilizada nos cenários estudados. Destacou-se também que a utilização de plataformas online é bastante inferior para os premiados de categorias iniciantes em comparativo aos avançados. O estudo evidenciou ainda que os premiados em geral possuem habilidades em matemática e algoritmos, domínios essenciais para o sucesso na competição.

Utilizando as questões abordadas, foi possível identificar características comuns para os premiados das olimpíadas a partir dos perfis de competidores analisados, assim, a partir dos dados obtidos pelo estudo, o competidor da OPI pode avaliar quais aspectos e atitudes tomadas pelos competidores com melhores desempenhos, e a partir disso, julgar características necessárias para melhorar seu desempenho.

\section{Agradecimentos}

Gostaríamos de agradecer aos membros do Projeto Olímpico da UFCG, pelo apoio concedido para realização da pesquisa que gerou este artigo como artefato.

\section{Referências}

[Burton 2008] Burton, B. A. (2008). Breaking the routine: Events to complement informatics olympiad training. volume 2, pages 5-15. Olympiads in Informatics.

[da Silva Santos et al. 2015] da Silva Santos, E. F., Pedrosa, V. M. D., da Silva Guedes, J. F., and Miranda, M. V. C. (2015). Olimpíadas científicas como ferramenta de difusão do conhecimento e aprendizagem aos alunos do município de Areia/PB. Congresso Nacional de Educação (II Conedu).

[Dagienè 2010] Dagienè, V. (2010). Sustaining informatics education by contests. In Proceedings of the 4th International Conference on Informatics in Secondary Schools Evolution and Perspectives: Teaching Fundamentals Concepts of Informatics, pages 1-12. Springer-Verlag. 
VII Congresso Brasileiro de Informática na Educação (CBIE 2018)

Anais do XXIX Simpósio Brasileiro de Informática na Educação (SBIE 2018)

[de Barros et al. 2009] de Barros, L. G., Ribeiro, S. P. S., and Oeiras, J. Y. Y. (2009). Projeto de extensão universitária para apoio e realização da Olimpíada Brasileira de Informática em escolas. XXIX Congresso da SBC-XVII WEI.

[do Nascimento et al. 2007] do Nascimento, M. G., Palhano, D., and Oeiras, J. Y. Y. (2007). Competições escolares: uma alternativa na busca pela qualidade em educação. pages 284-287. Simpósio Brasileiro de Informática na Educação-SBIE.

[Duarte et al. 2010] Duarte, A., Moreira, H., and Mello, T. S. (2010). Competitividade como fator motivacional para o estudo de computação. Simpósio Brasileiro de Informática na Educação-SBIE.

[Fayyad et al. 1996] Fayyad, U., Piatetsky-Shapiro, G., and Smyth, P. (1996). The KDD process for extracting useful knowledge from volumes of data. pages 27-34. Communications of the ACM, 39(11).

[Glaser and Strauss 1999] Glaser, B. G. and Strauss, A. L. (1999). Discovery of Grounded Theory Strategies for Qualitative Research. Routledge, 1st edition.

[ICPC 2018] ICPC (2018). ACM-ICPC International Collegiate Programming Contest. https: / / icpc. baylor. edu/. Acessado em 30 de junho de 2018.

[Judge 2018] Judge, U. O. (2018). URI Online Judge. https://www . urionline judge. com. br/. Acessado em 30 de junho de 2018.

[Kiryukhin 2010] Kiryukhin, V. M. (2010). Mutual influence of the national educational standard and olympiad in informatics contents. volume 4, pages 15-29. Olympiads in Informatics.

[OBI 2018] OBI (2018). Olimpíada Brasileira de Informática. https : / / ol impiada . ic.uni camp.br/. Acessado em 30 de junho de 2018.

[OPI 2018] OPI (2018). Olimpíada Paraibana de Informática. http://www. dsc . ufcg.br/ opi. Acessado em 30 de junho de 2018.

[Rocha et al. 2016] Rocha, T. O., Carvalho, E. V., de Oliveira Carvalho, L., Amaral, C. E. S., and Santos, P. A. (2016). As olimpíadas científicas no desenvolvimento da educação brasileira. Congresso Nacional de Educação (III Conedu). 